\title{
Rauchersprechstunde als Intervention zur Tabakentwöhnung bei stationären Patienten - Ergebnisse einer Pilotstudie
}

\section{Smoking-Cessation-Counselling as a Smoking Cessation Interventional Tool for Hospitalized Patients - Results of a Pilot Study}

Autor

Institut

\section{Bösch}

Zentrum für Pneumologie (Prof. Dr. med. T. Schaberg), Diakoniekrankenhaus Rotenburg (Wümme) eingereicht 1.6 .2009

akzeptiert nach Revision

17.6. 2009

\section{Bibliografie}

DOI $10.1055 / \mathrm{s}-0029-1214904$

Pneumologie 2009; 63:

578-581 @ Georg Thieme

Verlag KG Stuttgart · New York ISSN 0934-8387

\section{Korrespondenzadresse}

Dr. med. Dennis Bösch

Zentrum für Pneumologie

Diakoniekrankenhaus Roten-

burg

Verdener Straße 200

27357 Rotenburg (Wümme)

Boesch@ATEM-Online.de

\section{Zusammenfassung \\ V}

Hintergrund und Fragestellung: Der Anteil der rauchenden Bevölkerung ist hoch. Hiermit einhergehend findet sich ein hoher Anteil tabakrauchassoziierter Morbidität und Mortalität. Eine stationäre Klinikbehandlung ist prinzipiell eine günstige Situation, eine Tabakentwöhnung einzuleiten. Ziel dieser Arbeit war es, die Effektivität einer Raucherberatung, die im Rahmen eines stationären Aufenthaltes durchgeführt wurde, zu evaluieren.

Methodik: Evaluiert wurden 25 konsekutive Patienten, die das Angebot einer Raucherberatung im Rahmen ihres stationären Aufenthaltes in einer Lungenklinik annahmen. Verschiedene Daten zum Rauchverhalten und zur Morbidität wurden im Rahmen der qualifizierten Beratung erfasst. Den Patienten mit einem FNDT $\geq 3$ wurde eine pharmakologische Unterstützung angeboten. Das Rauchverhalten wurde erneut 6 Monate nach Entlassung über ein Telefoninterview erfasst und teilweise über Carboxy-Hämoglobin-Messungen validiert.

Ergebnisse: In den Tagen nach Beratung hatten 20 der 25 Patienten bzw. 80\% das Rauchen aufgegeben. Nach 6 Monaten waren insgesamt noch 11 Patienten abstinent, entsprechend einer Langzeiterfolgsrate von $44 \%$. Bei den Patienten mit medikamentöser Unterstützung lag der Anteil der Langzeitabstinenten um $55 \%$ höher als bei denen ohne medikamentöse Unterstützung.

Schlussfolgerung: Neben der Praktikabilität konnte die Effektivität einer qualifizierten Raucherberatung, begleitend zur Behandlung stationärer Patienten gezeigt werden. Die Ergebnisse ermutigen zur weiteren Verbreitung und Anwendung dieser Interventionsmethode in unserem Gesundheitssystem.

\section{Abstract \\ V}

Background and objective: In perspective of a high prevalence of smokers in our population, we find a high proportion of smoking-related morbidity and mortality. Hospitalisation is basically a favourable situation to start smoking cessation. Aim of the study was to show the effectivity of smoking-cessation-counselling as a smoking cessation interventional tool for hospitalized patients.

Methods: We evaluated 25 consecutive patients, who had accepted an offer for smoking cessation counselling, while being hospitalized in a center of pulmonology. Data concerning smoking habits and morbidity had been collected in the course of a qualified smoking-cessation-counselling. Patients with a FNDT $\geq 3$ had been offered pharmacological support. The smoking behaviour had been re-collected 6 month after discharge via telephoninterview. Partly there was the opportunity to validate the information via measurement of carboxyhemoglobin.

Results: Following the smoking-cessation-counselling 20 out of 25 patients ( $80 \%$ ) had quit smoking. After 6 month 11 patients still had been abstinent, in accordance with a long-term-successrate of $44 \%$. Patients who accepted a pharmacological support had a 55\% higher long-termabstinence-rate.

Conclusion: Qualified smoking-cessation-counselling concomitantly to hospital treatment is feasible and effective. The results encourage distributing and administering this interventional tool in our health system. 


\section{Hintergrund und Fragestellung}

In Deutschland beträgt der Anteil der rauchenden Bevölkerung (15 Jahre und älter) etwa ein Drittel [1]. Unstrittig ist, dass unzählige Erkrankungen durch Zigarettenrauchen verursacht oder begünstigt sind [2]. Die hiermit assoziierte Morbidität und Mortalität führt dazu, dass ca. $22 \%$ aller Todesfälle bei den Männern und ca. 5\% bei den Frauen (bzw. ca. 140000 Todesfälle/Jahr) hierzulande zu Lasten des Rauchens gehen [3]. Nicht zuletzt aufgrund der Assoziation von Rauchen und allgemeiner Morbidität ergibt sich ein hoher Anteil rauchender Patienten im stationären Alltag der Kliniken. Je nach Schwerpunkt der Abteilung variiert der Anteil zwischen 25 und 55\% [4,5]. Im eigenen Patientengut ergab sich ein Anteil von ca. 30\%. Aus vielerlei Hinsicht wäre es wünschenswert, dass entsprechende Patienten im Rahmen ihres stationären Aufenthaltes (zusätzlich bzw. begleitend) eine Tabakentwöhnung durchführen würden. Bedingt durch die spezielle Situation während eines stationären Aufenthaltes ergeben sich zumeist einige Vorteile hinsichtlich einer erhöhten Effektivität einer Tabakentwöhnung im Vergleich zu ambulanten Patienten. Die neuen gesetzlichen Rahmenbedingungen (rauchfreie Krankenhäuser) sind hierbei nur ein begünstigender Faktor.

Die meisten Raucher haben den Wunsch, mit dem Rauchen aufzuhören. Aus Umfragen geht hervor, dass ca. 70\% aller Raucher das Rauchen prinzipiell aufgeben wollen und ca. $45 \%$ einen entsprechenden Versuch in den letzten 12 Monaten unternommen haben [6]. Andererseits liegen die langfristigen Erfolgsaussichten bei Spontanversuchen ohne spezielle Unterstützung nur bei etwa $5 \%$ [7]. Vielfach wurde gezeigt, dass bereits kurze beratende Gespräche den Erfolg signifikant steigern können. Die besten Langzeitergebnisse lassen sich jedoch durch längerfristig angelegte verhaltenstherapeutische und ggf. zusätzliche medikamentöse Unterstützung erzielen $[8,9]$.

Für Raucher, die (aus diversen Gründen) eine stationäre Behandlung benötigen, ergibt sich eine besondere Situation. Zum einen ist das Fortsetzen der Rauchgewohnheiten durch den veränderten Tagesablauf in der Klinik meist deutlich gestört, zum anderen ist das Rauchen aufgrund von Bettlägerigkeit bzw. eingeschränkter Mobilität oder Auflagen zum Rauchverhalten seitens des Krankenhauses zum Teil deutlich erschwert. Hinzu kommt, dass einige Patienten auch aufgrund ihres eingeschränkten gesundheitlichen Zustands das Rauchen (zumindest vorübergehend) einschränken oder aufgeben müssen. Ist die für die stationäre Behandlung zugrundeliegende Erkrankung zudem mit dem Zigarettenrauchen assoziiert, liegt eine gesonderte Situation vor, die zugunsten einer Erhöhung der Motivation zur Rauchaufgabe genutzt werden kann und sollte.

Zusammenfassend kann davon ausgegangen werden, dass die Situation während eines stationären Krankenhausaufenthaltes zur Einleitung einer Rauchaufgabe bzw. Tabakentwöhnung günstig ist. Ziel dieser Arbeit war es, die Effektivität bzw. den langfristigen Erfolg einer Raucherberatung, die im Rahmen eines stationären Aufenthaltes durchgeführt wurde, zu evaluieren.

\section{Methodik}

$\nabla$

Im Zeitraum zwischen 01/2008 und 08/2008 wurden 25 konsekutive Patienten, die das Angebot einer Raucherberatung/ -sprechstunde während ihres Krankenhausaufenthaltes in einer Lungenklinik (Zentrum für Pneumologie, Diakoniekrankenhaus Rotenburg) angenommen hatten, evaluiert und nachbeobachtet.
Den Patienten wurde die spezielle Beratung, unabhängig von der weiteren stationären Therapie, als offenes Angebot im Rahmen des Aufnahmegesprächs oder bei den nachfolgenden Visiten dargelegt. Bei Interesse waren die Patienten aufgefordert, einige wenige Fragen zu den Rauchgewohnheiten sowie den Fagerström-Test für Nikotinabhängigkeit (FTND) schriftlich zu beantworten. Der vermittelnde Stationsarzt wurde zudem gebeten, grundlegende Informationen zur Lungenfunktion, laufenden Medikation und zur Komorbidität zu ergänzen. Die Durchführung der Raucherberatung wurde von einem für Tabakentwöhnung speziell qualifizierten Facharzt der Klinik durchgeführt und dauerte jeweils 25 bis 30 Minuten. Im Rahmen längerer stationärer Aufenthalte waren in Einzelfällen weitere einzelne Kurzkontakte von 2-3 Minuten durchgeführt worden. Inhalt des Beratungsgesprächs war: Steigerung der Motivation, Aufdecken persönlicher Verhaltensmuster, Erarbeiten eines individuellen Plans incl. Vorbereitung auf mögliche Schwierigkeiten und Umgang mit dem sozialen Umfeld. Bei einem FTND $\geq 3$ wurde zudem die Möglichkeit einer Nikotinersatztherapie in Form eines Nikotinpflasters angeboten und der Patient entsprechend aufgeklärt.

Die Erhebung der Basisdaten erfolgte unmittelbar vor bzw. während der Beratung. Die Effektivität der Intervention wurde jeweils 6 Monate später über ein kurzes Telefoninterview ermittelt. Für einige Patienten konnten aus den Patientenakten zusätzlich Blutgasanalysedaten aus Folgekontakten (5-7 Monate nach Entlassung) zum Vergleich (Kontrolle über den Carboxy-Hämoglobin-Anteil, $\mathrm{COHb}$ ) herangezogen werden.

Die statistische Analyse erfolgte nach Anonymisierung der Daten. Die Auswertung erfolgte deskriptiv und wurde im Weiteren explorativ beurteilt. Die Analyse erfolgte mithilfe von StatView for Windows (Version 5.0).

\section{Ergebnisse}

\section{$\nabla$}

Es wurden insgesamt 25 Patienten in die Beobachtung eingeschlossen und nach 6 Monaten erneut befragt. Eine Patientin war in der Zwischenzeit verstorben, gehörte jedoch zu denen, die das Rauchen nicht aufgegeben hatten. Das Alter der Patienten $(n=25)$ lag zwischen 32 und 73 Jahren. Die Geschlechtsverteilung war ausgeglichen: 13 Männer und 12 Frauen. Die Aufnahmediagnosen waren überwiegend zigarettenrauchassoziiert (22 x COPD (GOLD I bis IV), $2 \times$ Bronchial-Carcinom, $1 \times$ Asthma bronchiale). Die Basisdaten der Patienten sind in $\bullet$ Tab. 1 zusammengefasst.

Aufgrund der Beratung haben 20 Patienten (80\%) das Rauchen vollständig eingestellt. Hiervon hatten 10 Patienten einem FTND $\geq 3$. Von diesen 10 Patienten haben 6 eine NET und 1 Patient Vareniclin unterstützend eingesetzt. 13 Patienten hatten keine medikamentöse Unterstützung. Nach 6 Monaten waren insgesamt noch 11 Patienten bzw. 55\% der 20 Patienten, die das Rauchen

Tab. 1 Basisdaten der 25 Patienten (Alter zum Beratungszeitpunkt, durchschnittlicher Zigarettenkonsum in den letzten Wochen, gerauchte Packungsjahre, Punktwert des FTND, Alter bei Beginn des Rauchens)

\begin{tabular}{|c|c|c|}
\hline & Mittelwert (SD) & Range \\
\hline Alter & $55,8(10,2)$ & $32-73$ \\
\hline Zigaretten proTag & $16,9(8,1)$ & $4-36$ \\
\hline Packungsjahre & $44,1(15,0)$ & $20-90$ \\
\hline FTND & $3,3(2,2)$ & $1-9$ \\
\hline Alter bei Beginn & $18,2(1,8)$ & $13-22$ \\
\hline
\end{tabular}




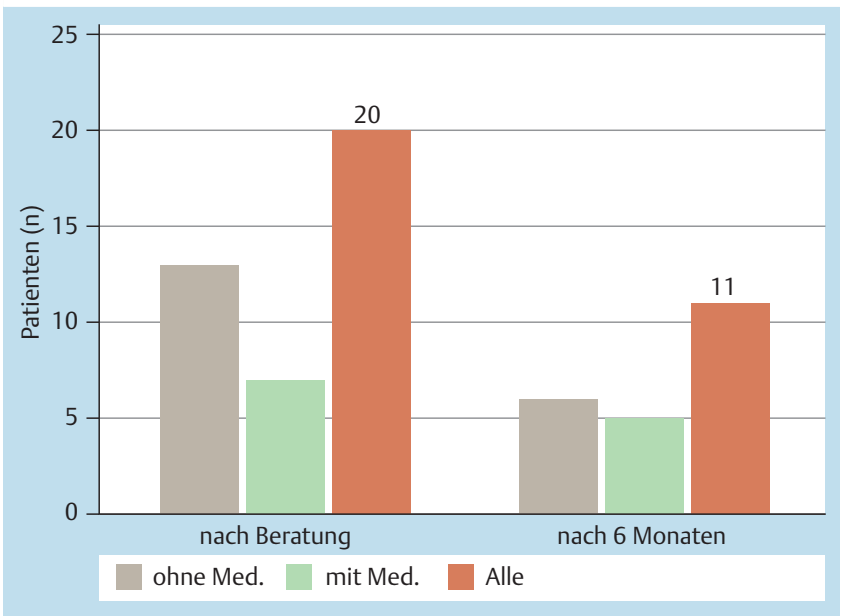

Abb. 1 Abstinenzraten der 25 Patienten, gesamt und jeweils ohne und mit medikamentöser Unterstützung, nach Beratung bzw. nach 6 Monaten.

nach der Beratung aufgegeben hatten, abstinent. Somit ergab sich letztlich ein Langzeiterfolg von 44\% bzw. 11 der 25 Patienten (s. - Abb. 1). Betrachtet man die Gruppe der 20 Patienten, die das Rauchen aufgegeben haben, ergibt sich folgendes Bild bzgl. des Erreichens einer Langzeitabstinenz. Bei den 7 Patienten mit medikamentöser Unterstützung lag der Anteil der Langzeitabstinenten bei 5 bzw. 71\% im Vergleich zu 6 von 13 Patienten (bzw. 46\%) ohne medikamentöse Unterstützung.

Die Information des Telefoninterviews bzgl. der Langzeitabstinenz konnte bei den 12 Patienten, die angaben, weiterhin abstinent zu sein, teilweise biometrisch kontrolliert werden. Bei 6 Patienten wurde ein niedriger $\mathrm{COHb}(<2 \%)$ gemessen, der die Abstinenz bestätigte. Ein Patient hatte, bei deutlich erhöhtem $\mathrm{COHb}$, offensichtlich eine Falschangabe gemacht und bei 5 Patienten standen keine biometrischen Kontrolldaten zu Verfügung.

\section{Diskussion und Schlussfolgerung \\ $\nabla$}

In der Studie wurden Patienten, denen eine Beratung zur Tabakentwöhnung im Rahmen ihres stationären Aufenthaltes angeboten wurde, beobachtet und evaluiert. Die Ergebnisse der Arbeit zeigen, dass eine stationäre Tabakentwöhnung bei motivierten Patienten praktikabel und effektiv ist. Zweitens bestätigt sich, dass eine zusätzliche Nikotinersatztherapie bzw. medikamentöse Unterstützung die Effektivität bzw. den Langzeiterfolg verbessert.

Die Tabakentwöhnung ist in Anbetracht der enormen gesundheitlichen und sozioökonomischen Folgen des Zigarettenrauchens eine der wichtigsten präventiven und therapeutischen Maßnahmen in der Medizin. Für die Volkskrankheit COPD zum Beispiel gilt die Tabakentwöhnung als die wichtigste therapeutische Einzelmaßnahme überhaupt [10].

Dennoch wird die Tabakentwöhnung im Deutschen Gesundheitssystem unzureichend berücksichtigt [11]. Zudem ist die Tabakentwöhnung eine schwierige Herausforderung. Die Versagerquote ist hoch und entsprechende Frustrationen nicht selten. Die langfristige Erfolgsquote eines Spontanversuchs ohne spezielle Unterstützung liegt lediglich bei ca. 5\% [7]. Um die Zahl der Rauchaufgaben zu erhöhen und die Rate der erfolgreichen Tabakentwöhnungen zu steigern, ist es zum einem notwendig, die Anzahl der Entwöhnungsversuche zu erhöhen und andererseits die
Erfolgsquote des einzelnen Versuchs zu verbessern. Es ist allgemein gefordert, dass möglichst bei jedem Arztbesuch bzw. -kontakt die Rauchgewohnheiten erfasst und die Möglichkeiten einer Tabakentwöhnung eruiert und gefördert werden. Aus multiplen Gründen ist dieses Ideal jedoch fern der Realität. Dies ist auch für stationäre Patienten zutreffend. Für hospitalisierte Patienten gelten dabei besondere Bedingungen, die oftmals für eine verbesserte Erfolgsaussicht genutzt werden könnten. Es scheint daher in besonderem Maße sinnvoll zu versuchen, stationäre Patienten zur Rauchaufgabe zu motivieren und Hilfestellung für eine verbesserte Erfolgsaussicht einer Tabakentwöhnung zu geben. Ein Review der Cochrane Collaboration [12] kam zu dem Schluss, dass Raucherentwöhnungsprogramme, die während eines Krankenhausaufenthaltes, unabhängig von der Diagnose, begonnen und mit unterstützenden Kontakten für mindestens einen Monat nach Entlassung fortgesetzt wurden, effektiv sind. Patienten mit rauchassoziierten Erkrankungen seien zudem in besonderem Maße empfänglich für eine entsprechende Hilfe.

Die vorliegende Arbeit zeigt die Ergebnisse einer speziellen Intervention, die sicher über das normale Maß einer Beratung im stationären Setting hinausgeht, andererseits jedoch bzgl. des Zeitaufwandes im Vergleich zu anderen professionellen Angeboten zur Tabakentwöhnung als moderat einzustufen ist. Die beobachtete Langzeitabstinenzrate nach 6 Monaten lag mit 48\% vergleichsweise hoch. Ergebnisse der Lung Health Study von 5887 COPD-Patienten ergaben eine 12-Monats-Abstinenzrate in den Interventionsgruppen (12 Gruppensitzungen und pharmakologische Unterstützung) von ca. 35\% [13]. Aufgrund der unterschiedlichen Beobachtungszeiträume, der verschiedenen Ausgangsvoraussetzungen und einem heterogeneren Patientengut sind die Daten nur bedingt vergleichbar. COPD-Patienten haben meist eine höhere Tabakabhängigkeit und gelten als schwer-entwöhnbar. Bei der Beurteilung der vorliegenden Daten sind einige weitere Dinge zu berücksichtigen. Das Angebot galt für Patienten, die bereits ein hohes Maß an Motivation hatten und nicht überredet werden mussten. Es handelte sich überwiegend um Patienten mit rauchassoziierten Erkrankungen, denen der Zusammenhang zwischen Rauchen bzw. Nichtrauchen und dem Fortgang ihrer Erkrankung im Rahmen ihrer stationären Behandlung erläutert wurde und denen eine Tabakabstinenz als eine essenzielle Maßnahme nahegelegt wurde. Die Beratungsmaßnahme wurde durch einen speziell für eine Tabakentwöhnung qualifizierten und mit dieser Thematik erfahrenen Internisten durchgeführt. Das Angebot wurde seitens der Patienten überwiegend positiv aufgenommen und als Zeichen besonderer Fürsorge bewertet. Selbstkritisch ergeben sich für die Studie einige Limitationen. Die Fallzahl dieser Beobachtungsstudie war gering. Eine Validierung sollte ergänzend mit einer größeren Patientenzahl durchgeführt werden. Eine nach oben verfälschte Abstinenzrate in der Nachbeobachtung nach 6 Monaten konnte, zumindest bei einem Teil der Patienten, aufgrund der Methode (Telefoninterview) nicht ausgeschlossen werden. Wünschenswert wäre die Möglichkeit eines standardisierten biometrischen Kontrollverfahrens (z. B. COHbBestimmung) bei allen Patienten gewesen.

Patienten mit einem FNDT von $\geq 3$ Punkten wurde eine Nikotinersatztherapie (NET) angeboten. Eine NET zielt auf eine Verminderung der Entzugssymptomatik sowie des Rauchverlangens. Ein Cochrane Metaanalyse zu diesem Thema ergab eine Verbesserung der langfristigen Abstinenzquote mithilfe einer NET um 50-70\% [14]. Dies lässt sich durch die vorliegenden Daten bestätigen. Die Patienten, die das Rauchen aufgaben, hatten mit pharmakologischer Unterstützung eine um $55 \%$ höhere Langzeit- 
abstinenzrate als die Patienten ohne pharmakologische Unterstützung. In der zwischenzeitlich publizierten Leitlinie zur Tabakentwöhnung bei COPD wird die kombinierte medikamentöse und psychosoziale Unterstützung für COPD-Patienten ausdrücklich empfohlen [11].

Zusammenfassend kann festgestellt werden, dass eine Beratung zur Tabakentwöhnung bei stationären internistischen Patienten praktikabel ist und sich in den Ablauf eines stationären Aufenthaltes einbetten lässt. Des Weiteren ergibt sich eine gute Effektivität der speziellen, vom Zeitaufwand vergleichsweise moderaten Maßnahme bei motivierten Patienten mit überwiegend tabakrauchassoziierten pulmonalen Grunderkrankungen. Die Ergebnisse berechtigen zudem zu der Hoffnung, dass entsprechende Interventionen in größerem Umfang den stationären Patienten unseres Gesundheitssystems angeboten werden können.

\section{Interessenkonflikte}

Kein Interessenkonflikt

\section{Literatur}

1 Statistisches Bundesamt (Hrsg). Leben in Deutschland - Ergebnisse des Mikrozensus 2005. Wiesbaden: 2006

2 U.S. Department of Health and Human Services, Centers for Disease Control and Prevention, National Center for Chronic Disease Prevention and Health Promotion. The Health Consequences of Smoking. A Report for the Surgeon General. Atlanta, GA: Office on Smoking and Health, 2004
3 Welte R, König HH, Leidl R. The Costs of Health Damage and Productivity Losses Attributable to Cigarette Smoking in Germany. Eur J Public Health 2000; 10: $31-38$

4 Rigotti NA, Arnsten JH, McKool KM, Wood-Reid KM, Pasternak RC, Singer $D E$. Smoking by patients in a smoke-free hospital: prevalence, predictors, and implications. Prev Med 2000; 31: 159-166

5 Smith J, O'Callaghan C. Exploration of in-patient attitudes towards smoking within a large mental health trust. Psychiatric Bulletin 2008; 32: $166-169$

6 Centers for Disease Control and Prevention. Cigarette Smoking among Adults - United States, 1995. Morb Mortal Wkly Rep 1997; 46: 1217 1220

7 Hughes JR. New Treatments for Smoking Cessation. CA Cancer J Clin 2000; 50: $143-151$

8 Nardini S. Smoking Cessation. Eur Respir Mon 2008; 42

9 Batra A, Buchkremer G. Tabakentwöhnung - Ein Leitfaden für Therapeuten. Stuttgart: Kohlhammer, 2004

10 Vogelmeier C, Buhl R, Criée CP et al. Leitlinie der Deutschen Atemwegsliga und der Deutschen Gesellschaft für Pneumologie und Beatmungsmedizin zur Diagnostik und Therapie von Patienten mit chronisch obstruktiver Bronchitis und Lungenemphysem (COPD). Pneumologie 2007; 61: e1 - e40

11 Andreas S, Batra A, Behr J et al. Tabakentwöhnung bei COPD. S3 Leitlinie herausgegeben von der Deutschen Gesellschaft für Pneumologie und Beatmungsmedizin. Pneumologie 2008; 62: 255-272

12 Rigotti N, Munafo MR, Stead LF. Interventions for smoking cessation in hospitalised patients. Cochrane Database of Systematic Reviews 2007, Issue 3

13 Anthonisen NR, Connett JE, Kiley JP et al. Effects of smoking intervention and the use of an inhaled anticholinergic bronchodilator on the rate of decline of FEV1. The Lung Health Study. JAMA 1994; 272: 1497-1505

14 Stead LF, Perera R, Bullen C, Mant D, Lancaster T. Nicotine replacement therapy for smoking cessation. Cochrane Database of Systematic Reviews 2008, Issue 1 